\title{
Selection of Appropriate Detection Scheme for Optimum Performance-Complexity Trade-Off in 3GPP Suburban Macrocell Wireless MIMO Environments
}

\author{
Nikita Jain \\ Student, M.Tech \\ Department of Electronics and \\ Communication Engineering \\ S.S College of Engineering \\ Udaipur, Rajasthan
}

\author{
Shivpratap Pandey \\ Assistant Professor \\ Department of Electronics and \\ Communication Engineering \\ S.S College of Engineering \\ Udaipur, Rajasthan
}

\author{
Piyush Sharma \\ Assistant Professor \\ Department of Electrical \\ Engineering \\ S.S College of Engineering \\ Udaipur, Rajasthan
}

\begin{abstract}
This paper presents an analytical solution to the problem of selecting an optimum symbol detector for wireless MIMO communications in the LTE/4G suburban macrocell environments. Since, the use of a symbol detector in MIMO systems is often limited by the complexity it offers, it is of vital importance to use a detector which offers reliable performance but does not offer high complexity. This paper analyses the error rate performance and computational complexity of Zero Forcing (ZF), Minimum Mean Square Error (MMSE) and Maximum Likelihood (ML) and suggests the optimum detectors with reasonable complexityperformance trade-off.
\end{abstract}

\section{Keywords}

MIMO, Symbol detector.

\section{INTRODUCTION}

Multiple-input-multiple-output (MIMO) technology has potential to greatly enhance the capacity of wireless cellular networks and/or the reliability of data transmission through wireless media [1]. Upcoming new standards, such as 3GPP LTE-Advanced and 802.11ac, are exploiting large MIMO systems to push the data rates in consumer wireless communications [2]. To fully exploit the potential of MIMO, a high- fidelity and low-complexity detection scheme at the receiving end is needed.

In spatial multiplexing, the optimum detection technique is Maximum-Likelihood (ML) detection which is exhaustive in nature i.e. it performs search operation over entire symbol set [3]. This leads to exponential increase in complexity with increase in number of transmit antenna and modulation order and thus makes it impractical to be used for higher order constellations $[2,3]$.

To avoid such highly complex hardware, sub optimal detection algorithms such as Zero Forcing (ZF) [4] and Minimum Mean Square Error (MMSE) [5] are used. These. Algorithms offer very less complexity and are often used in practical MIMO hardware in one form or other.

Rest of this paper is divided into 6 sections. Section 2 explains the MIMO system model. Section 3 gives a detailed explanation of ZF, MMSE and ML detector. Section 4 presents complexity analysis of ZF and MMSE detectors. Section 5 contains the specifications and scenario of 3GPP suburban macrocell environment. Section 6 presents the results and discussion. Section 7 concludes the paper.

\section{MIMO SYSTEM MODEL}

A MIMO system with 'MT' transmit antenna and 'MR' receiving antenna is represented as [6]

$\mathrm{y}=\mathrm{Hs}+\mathrm{n}$

Where $\mathrm{s}$ is [MT $\mathrm{x}$ 1] transmitted symbol vector derived, $\mathrm{y}$ is received symbol vector of dimension [MR $\mathrm{x} 1], \mathrm{H}$ is [MR $\mathrm{x}$ MT] channel matrix and $n$ is [MR $x$ 1] dimensional noise vector introduced by channel.

\section{CONVENTIONAL DETECTION METHODS}

On the basis of error rate performance, symbol detection methods are classified as optimal, sub optimal and near optimal algorithms [7]. Maximum Likelihood (ML) detection is offers optimum performance but it uses exhaustive search approach which leads to very high complexity [8]. Suboptimal algorithms exhibit very low complexity but unlike ML detection, their performance is not reliable for bad channels. Commonly used sub optimal methods are Zero Forcing (ZF), Minimum Mean Square Error (MMSE) Method and Successive Interference Cancellation (SIC) $[8,9]$. Near optimal detection methods are generally better performing with comparatively lower complexity than ML, but they are still evolving as their hardware implementation is a tedious task [10-12].

\subsection{Maximum Likelihood (ML) Detection}

ML detection aims at minimizing the noise. The ML data vector is derived from equation (1) as.ML detection is carried out by searching for all the candidate vectors from the transmitting symbol set exhaustively and then selecting the one with the smallest error from received symbol. Complexity of ML detection increases exponentially with the number of transmitted antennas because it searches for every possible candidate. For example, when a transmitter is equipped with 4antennas and 4-QAM scheme is used, total number of possible candidates is $44=256$.

$\widehat{\mathrm{SL}_{\mathrm{ML}}}=\arg \min _{\mathrm{s} \in|s|^{N_{\mathrm{T}}}}\|y-\mathrm{Hs}\|^{2}(2)$

\subsection{Equalization Based Detection}

In linear equalization based detection, an estimate of the transmitted data vectors is formed using an equalization matrix ' $G$ ' [2]. 


\subsubsection{Zero Forcing $(Z F)$ Detection}

The equalization matrix ' $G$ ' for $Z F$ detection method is given by the pseudo-inverse of $\mathrm{H}[5]$

$$
G_{Z F}=H^{\#}=\left(H^{H} H\right)^{-1} H^{H}
$$

Thus, the result of ZF equalization is

$$
\widehat{G_{Z F}}=G_{Z F} y=\left(H^{H} H\right)^{-1} H^{H} y=s+\tilde{n}
$$

Which is the transmitted data vector s corrupted by the transformed noiseñ $=\mathrm{H}^{\#} \mathrm{n}$. This means that the interference caused by the channel $\mathrm{H}$ is completely removed "Forced to zero". However, in general the transformed noise $\tilde{n}=H^{\#} n$ is larger than $n$ i.e. noise enhancement [5]. As can be seen from equation (4), the complexity of ZeroForcing detector is linear, but its performance can be unreliable in many cases.

\subsubsection{Minimum Mean Square Error (MMSE) \\ Detection}

To minimize the effect of environmental noise, the MMSE detector employs an equalizer which tries to nullify the noise by using noise variance at receiver. The equalization matrix ' $\mathrm{G}$ ' for MMSE is given by [5].

$\mathrm{G}=\left(\mathrm{H}^{\mathrm{H}} \mathrm{H}+\sigma_{\mathrm{W}}^{2} \mathrm{I}\right)^{-1} \mathrm{H}^{\mathrm{H}}$

Here $\mathrm{G}$ is minimizing the mean-square error. Thus, the result of MMSE equalization is

$$
y_{M M S E}=\left(H^{H} H+\sigma_{W}^{2} I\right)^{-1} y
$$

Like ZF detector, the complexity of MMSE is linear but ZF or MMSE detection can only exploit a diversity of order $r-t+1$ [5].

\section{COMPLEXITY OF EQUALIZATION BASED DETECTION METHODS}

The Zero Forcing technique is based on calculation of the pseudo-inverse of the channel matrix $\mathrm{H}$ of dimension $(\mathrm{r} \times \mathrm{t})$

The payload processing for ZF consists of a matrix-vector multiplication per transmitted vector and a slicing step to translate the estimated elements of $\mathrm{x}$ to the possible transmitted symbols. The matrix-vector multiplication is given by [6]

$$
\mathrm{H}^{\mathrm{P}}=\left(\mathrm{H}^{\mathrm{H}} \mathrm{H}\right)^{-1} \mathrm{H}^{\mathrm{H}}
$$

The complexity of this product is equal to MT2 (MR-1) complex additions and MT $\times$ MR complex multiplications. The result is a square matrix with dimension MT $\times$ MT. For this square matrix $\mathrm{HHH}$ the inverse needs to be determined. It was shown in [6], that the direct inversion of a given square matrix A (with dimension MR $\times$ MR) has a complexity in the order of MR3 additions andMR3 multiplications in total. So, inverting HHH has a complexity of MT3.

Finally, the inverse of $\mathrm{HHH}$ is multiplied by $\mathrm{HH}$. The complexity of this last multiplication is equal to MR addition and MT2 $\times$ MR multiplication. The payload processing for ZF consists of a matrix-vector multiplication per transmitted vector and a slicing step to translate the estimated elements of $\mathrm{s}$ to the possible transmitted symbols. The matrix-vector multiplication is given by [6]

$\widetilde{\mathrm{x}}=\mathrm{H}^{\mathrm{p}} \mathrm{r}$

The complexity of this product is equal to MT $\times(\mathrm{MR}-1)$ complex additions and MT $\times$ MR complex multiplications.

Summarizing, the complexity of the ZF algorithm per transmitted vector s equals [6]

$$
\begin{aligned}
C_{\mathrm{ZF}} \text { (flops) }= & 7 \mathrm{M}_{\mathrm{T}}^{3}+7 \mathrm{M}_{\mathrm{T}}^{2} \mathrm{M}_{\mathrm{R}}-2 \mathrm{M}_{\mathrm{T}}+4 \mathrm{M}_{\mathrm{T}} \mathrm{M}_{\mathrm{R}}+ \\
& \frac{1}{2} \mathrm{M}_{\mathrm{R}} \log _{2}(\mathrm{M})
\end{aligned}
$$

Complexity of MMSE

The complexity of the MMSE algorithm is almost equal to the complexity of the $\mathrm{ZF}$ method described in the previous section.

In the preamble-processing phase, the following MIMO processing matrix needs to be determined [6]

$$
\mathrm{G}=\left(\mathrm{H}^{\mathrm{H}} \mathrm{H}+\sigma \mathrm{I}_{\mathrm{M}_{\mathrm{T}}}\right)^{-1} \mathrm{H}^{\mathrm{H}}
$$

The calculation of this matrix has almost the same complexity as the determination of the pseudo-inverse in case of the $\mathrm{ZF}$ algorithm. Since is real, the only additional complexity consists of the real additions of $\sigma$ (i.e., the addition of $\sigma$ to the real part of the diagonal elements of $\mathrm{HH} \mathrm{H}$ ). This leads to a total complexity in the preamble-processing phase of

$$
\mathrm{C}_{\mathrm{MMSE}-\text { pre }} \text { (flops) }=7 \mathrm{M}_{\mathrm{T}}^{3}+7 \mathrm{M}_{\mathrm{T}}^{2} \mathrm{M}_{\mathrm{R}}
$$

The complexity of MMSE during the payload processing is equal to that of $\mathrm{ZF}$ and consists of a matrix-vector product

\section{3GPP SUBURBAN MACROCELL ENVIRONMENT}

The 3GPP Suburban Macrocell Environment represents sparsely populated environments with low infrastructure. Such type of environments generally correspond to villages, open areas, fields etc. Specifications for the environment are as follows [7]:

- Cell radius: up to $30 \mathrm{Kms}$

- Fading: Log Normal Shadow Fading

- Distance b/w transmitting antenna: $4 \lambda$

- Distance $\mathrm{b} / \mathrm{w}$ receiving antenna: $0.4 \lambda$

- Carrier frequency : $2 \mathrm{GHz}$

- Vehicular speed : 0Kmph/35Kmph/ 120Kmph

- Modulation order: 16-QAM/64-QAM/256-QAM

\section{RESULTS AND DISCUSSION}

The bit error rate performance of ZF, MMSE and ML detectors is measured on 3GPP suburban macrocell environment. Based upon the BER performance, optimum detector is decided. For the purpose of fair comparison, BER of 10-4 is taken as reference. All comparisons will be made on this BER.

Figure 1(a), 1(b) and 1(c) shows the BER performance of ZF, MMSE and ML detector with stationary user at 16-QAM, 64QAM and 256-QAM respectively.

Figure 1(a) reveals that for 16-QAM, ZF and MMSE perform very close to each other with ZF marginally outperforming MMSE. ML detector exhibits best performance amongst the three. ZF and MMSE achieve BER of 10-4 at nearly 8dB SNR while ML achieves the same at nearly 3dB SNR.

Figure 1(b) and 1(c) show similar trends in BER performance results. In both cases ZF marginally outperforms MMSE. For 64-QAM, BER of 10-4 is achieved at 9dB, for 256-QAM, it is 
nearly $11 \mathrm{~dB}$.

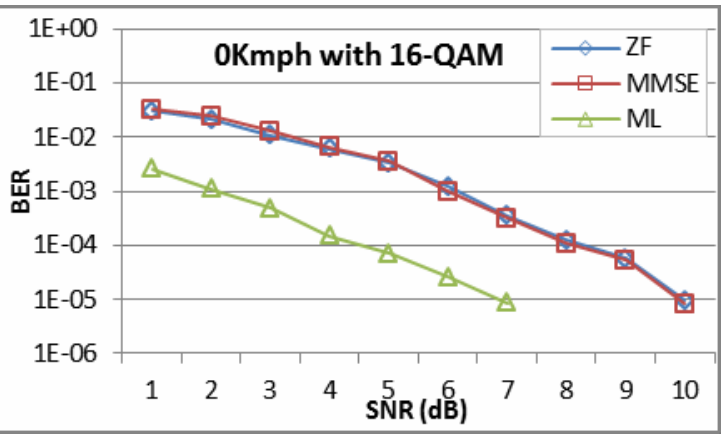

(a)

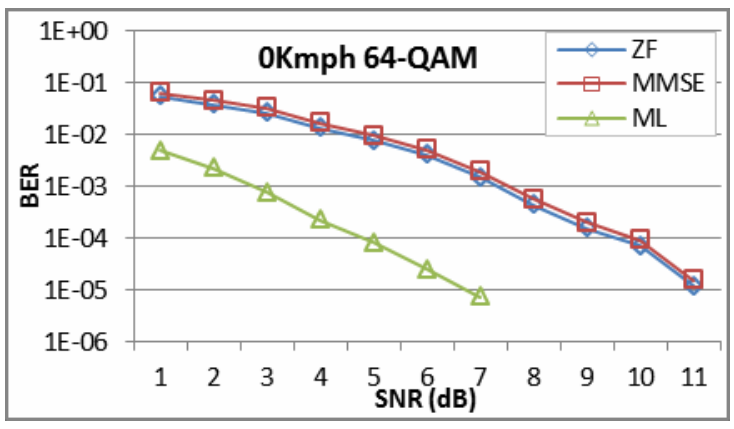

(b)

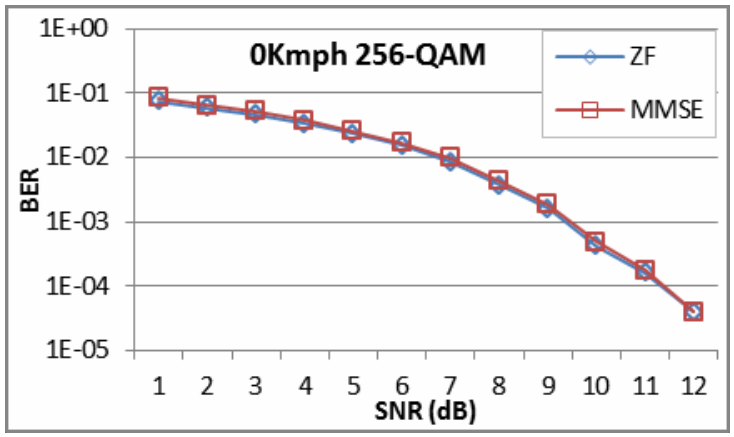

(c)

Figure 1: BER vs. SNR plots for stationary user using $4 \times 4$ MIMO with (a) 16-QAM (b) 64-QAM (c) 256-QAM

Figure 2 presents the error performance curves for mobile user velocity of $35 \mathrm{Kmph}$ with aforesaid modulation schemes.

It shows that increasing the velocity of end user increases the SNR requirements to maintain the error rate performance.

Figure 2(a) shows the BER vs. SNR plot for 16-QAM. It shows that ZF achieves BER of 10-4 at 8dB SNR followed by MMSE with minor SNR difference. ML detector achieves this BER at mere $4 \mathrm{~dB}$ SNR

For 64-QAM scheme, Figure 2(b) reveals that ZF and MMSE achieve the required BER of 10-4 at 9dB of SNR. ML, as usual, outperforms others and achieves zero BER at SNR of $8 \mathrm{~dB}$.

Figure 2(c) plots the BER vs. SNR curve ZF and MMSE detector with 256-QAM. At 12dB SNR, both these detectors achieve BER of 10-4.

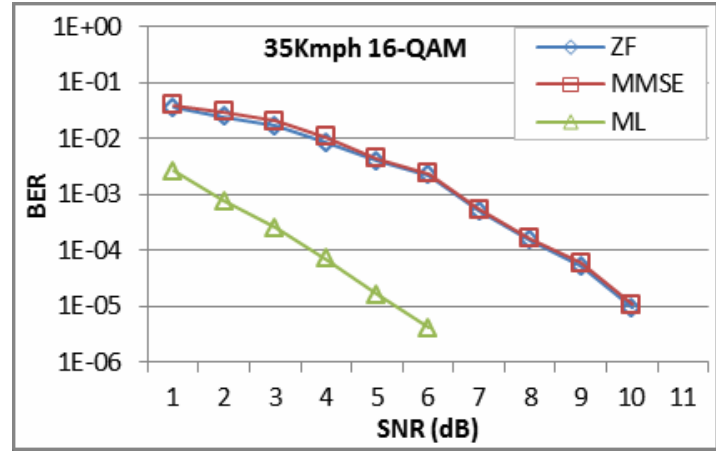

(a)

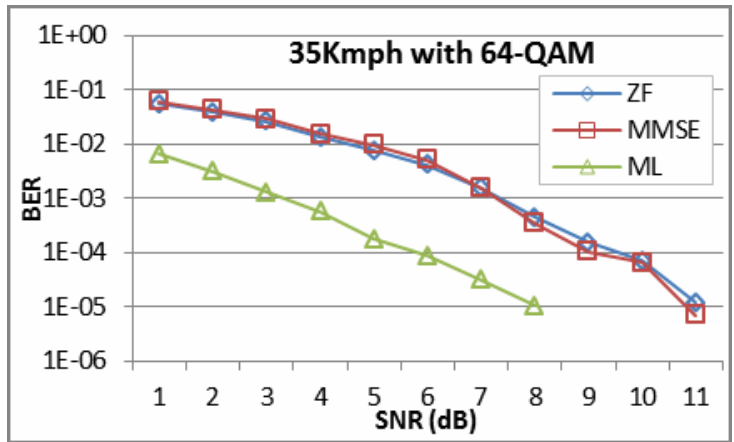

(b)

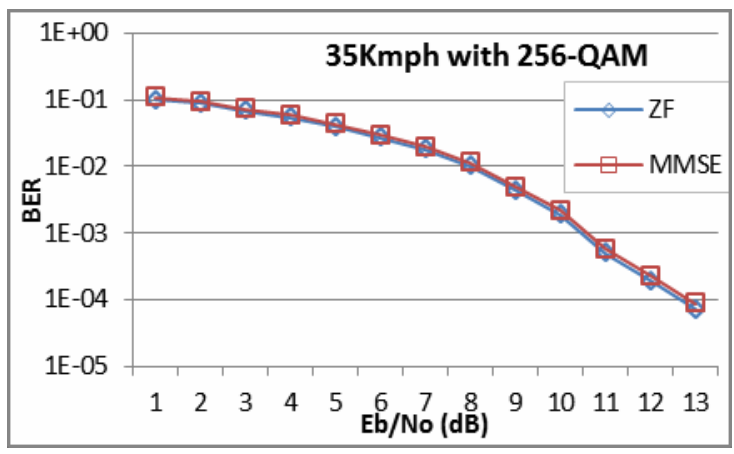

(c)

Figure 2: BER vs. SNR plots for user velocity of $35 \mathrm{Kmph}$ using 4x4 MIMO with (a) 16-QAM (b) 64-QAM (c) 256QAM

Figure 3(a) shows that for 16-QAM, ZF achieves BER of 10-4 at SNR of nearly $9.5 \mathrm{~dB}$ while MMSE achieves the same at $10.5 \mathrm{~dB}$. Throughout the measured SNR range, ZF and MMSE show performance difference of $1 \mathrm{~dB}$ SNR. ML achieves the same at about $6 \mathrm{~dB}$ SNR.

Figure 3(b) and 3(c) show BER vs. SNR plots for 64-QAM and 256-QAM. When using 64-QAM, ZF achieves the required BER at about $9.5 \mathrm{~dB}$ SNR which is achieved by MMSE at about $10 \mathrm{~dB}$

For 256-QAM, the same BER is achieved by ZF and MMSE at $12 \mathrm{~dB}$ and $13 \mathrm{~dB}$ respectively.

To summarize, ZF outperforms MMSE in every case, though the difference is substantial in case of end user speed of $120 \mathrm{Kmph}$. 


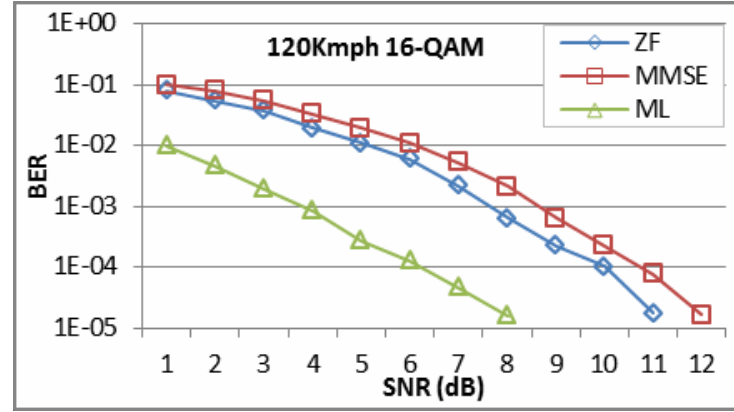

(a)

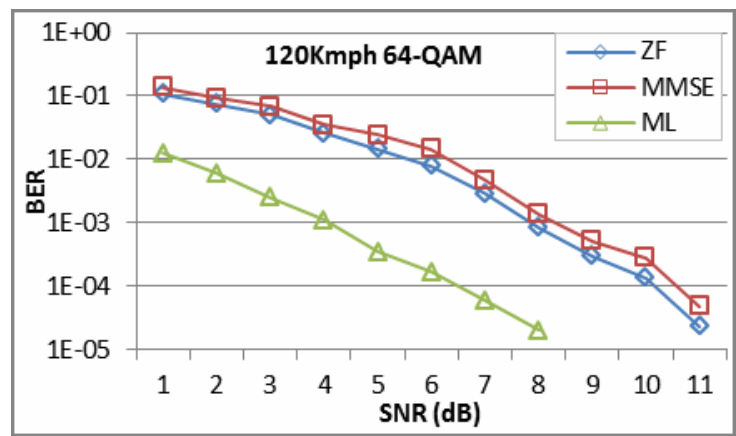

(b)

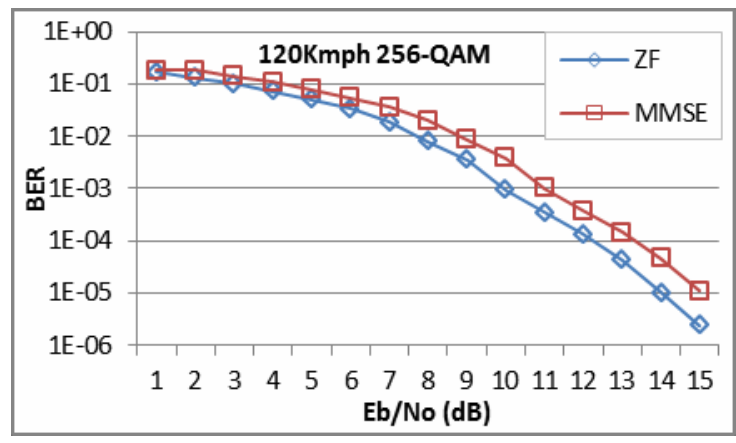

(c)

Figure 3: BER vs. SNR plots for user velocity of $120 \mathrm{Kmph}$ using 4x4 MIMO with (a) 16-QAM (b) 64-QAM (c) 256QAM

\section{CONCLUSION}

This paper presents a comparative analysis of error rate performance and complexity of MIMO symbol detectors to select optimum detector for 3GPP Suburban macrocell environments. Error rate performance results reveal that $\mathrm{ZF}$ detector performs better than MMSE for all modulation schemes with all kind of end user velocities. Also, complexity analysis reveals that ZF exhibits least complexity between the two detectors. It can therefore be concluded that ZF detector is optimum detector in terms of performance and complexity for 3GPP suburban macrocell environments. The results obtain via simulations will play a significant role in establishing the infrastructure for last mile connectivity in sub-urban and rural areas and will lead to improvement in Quality of Service (QoS).

\section{REFERENCES}

[1] R. Chang, and W. Chung, "Low -Complexity MIMO Detection Based on Post-Equalization Subspace Search," in IEEE transactions on Vehicular Technology, vol. 61, no. 1, January 2012

[2] K. Zhao, Y. Li, H. Jiang, and S. Du, in "A Low Complexity Fast Lattice Reduction Algorithm for MIMO Detection," in 2012 IEEE 23rd International Symposium on Personal, Indoor and Mobile Radio Communications (PIMRC).

[3] L. Bai and J. Choi, "Low Complexity MIMO detection," Springer.

[4] M. Jeon and J. Lee, "SNR Adaptive Limited Constellation Sphere Decoding," in IEEE conference onVehicular Technology, Fall 2009, Anchorage, pp. 1-5.

[5] D. Seethaler, H. Artes, and F. Hlawatsch, "Detection Techniques for MIMO Spatial Multiplexing Systems" ine\&i, vol. 122, no. 3, pp. 91-96, March 2005.

[6] A. M. Elshokry, "Complexity and Performance Evaluation of Detection Schemes for Spatial Multiplexing MIMO Systems", Thesis, IslamicUniversity, Gaza, January 2010.

[7] Spatial channel model for Multiple Input Multiple Output (MIMO) simulations. 3GPP http://www.3gpp.org/DynaReport/25996.htm

[8] Lai, G. Ascheid, H. Meyr, ,and T. Chiueh, Efficient Channel-Adaptive MIMO Detection Using JustAcceptable Error Ratell in IEEE Transactions on Wireless Communications, Vol. 10, issue 1, January 2011, pp. 73 83.

[9] K. Zhao, Y. Li, H. Jiang, And S. Du, -A Low Complexity Fast Lattice Reduction Algorithm For Mimo Detection,\| in 2012 IEEE 23rd International Symposium on Personal, Indoor and Mobile Radio Communications (PIMRC), pp. 1612-1616.

[10] C. Hung, W. Chung, R. Chang, and C. Chen, -A Monte Carlo MIMO Detection Scheme via Random Noise Generation, in IEEE Wireless Communications and Networking Conference (WCNC), 2012, April 2012, Shanghai, pp. 903-907.

[11]Lai, G. Ascheid, H. Meyr, ,and T. Chiueh, -Efficient Channel-Adaptive MIMO Detection Using JustAcceptable Error Ratell in IEEE Transactions on Wireless Communications, Vol. 10, issue 1, January 2011, pp. 73 83. 